\title{
Trichinella spiralis co-infection exacerbates Plasmodium berghei malaria-induced hepatopathy
}

Xu Mei ${ }^{1 \dagger}$, Zhanhong Ye ${ }^{2 \dagger}$, Yuqing Chang ${ }^{1}$, Shiguang Huang ${ }^{3 *}$, Jianping Song ${ }^{1 *}$ and Fangli Lu ${ }^{2^{*}}$

\begin{abstract}
Background: Although Plasmodium parasites and intestinal helminths share common endemic areas, the mechanisms of these co-infections on the host immune response remain not fully understood. Liver involvement in severe Plasmodium falciparum infections is a significant cause of morbidity and mortality. However, the effect of pre-existing Trichinella spiralis infection on the immune response and liver immune-pathogenesis in P. berghei ANKA (PbANKA)infected mice needs to be elucidated.
\end{abstract}

Methods: Outbred Kunming mice were infected with T. spiralis and 9 days later were challenged with $P$. berghei ANKA (PbANKA), and the investigation occurred at 13 days after co-infection.

Results: Compared with PbANKA-mono-infected mice, T. spiralis + PbANKA-co-infected mice had similar survival rate but lower PbANKA parasitaemia; however, there were more severe hepatosplenomegaly, increased liver and spleen indexes, and increased liver pathology observed by hematoxylin and eosin staining; higher expression levels of galectin (Gal)-1, Gal-3, CD68 ${ }^{+}$macrophages, and elastase-positive neutrophils measured by immunohistochemical staining; upregulated mRNA expression levels of Gal-1, Gal-3, cytokines (interferon-gamma (IFNY) and interleukin (IL)-6), and M1 macrophage polarization marker (inducible nitric oxide synthase (iNOS)) in the liver, and increased expression levels of Gal-1, IFNY, IL-6, eosinophil cationic protein, eosinophil protein X, and M1 (IL-1 $\beta$ and iNOS) and M2 (Ym1) macrophage polarization markers in the spleen of co-infected mice detected by using quantitative real-time reverse transcription polymerase chain reaction (qRT-PCR). In vitro study showed that compared with PbANKA-monoinfected mice, there were significantly increased expression levels of Gal-1, Gal-3, IL-6, IL-1 $\beta$, and iNOS in the peritoneal macrophage isolated from co-infected mice detected by using qRT-PCR. Correlation analysis revealed significant positive correlations between Gal-3 and IL-1 $\beta$ in the peritoneal macrophages isolated from PbANKA-mono-infected mice, between Gal-3 and IFNY in the spleen of co-infected mice, and between Gal-1 and Ym1 in the peritoneal macrophages isolated from co-infected mice.

\footnotetext{
*Correspondence: thshg@126.com; songjpgz@sina.com; fanglilu@yahoo.

com

${ }^{\dagger}$ Xu Mei and Zhanhong Ye contributed equally to this work

${ }^{1}$ Artemisinin Research Center and Institute of Science and Technology,

Guangzhou University of Chinese Medicine, Guangzhou, China

${ }^{2}$ Department of Parasitology, Zhongshan School of Medicine; Key

Laboratory of Tropical Disease Control of Ministry of Education, Sun Yat-

sen University, Guangzhou, China

${ }^{3}$ School of Stomatology, Jinan University, Guangzhou, China
}

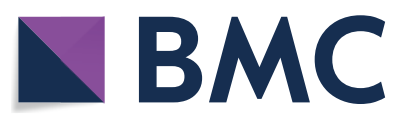

(c) The Author(s) 2020. This article is licensed under a Creative Commons Attribution 4.0 International License, which permits use, sharing, adaptation, distribution and reproduction in any medium or format, as long as you give appropriate credit to the original author(s) and the source, provide a link to the Creative Commons licence, and indicate if changes were made. The images or other third party material in this article are included in the article's Creative Commons licence, unless indicated otherwise in a credit line to the material. If material is not included in the article's Creative Commons licence and your intended use is not permitted by statutory regulation or exceeds the permitted use, you will need to obtain permission directly from the copyright holder. To view a copy of this licence, visit http://creativeco mmons.org/licenses/by/4.0/. The Creative Commons Public Domain Dedication waiver (http://creativecommons.org/publicdomain/ zero/1.0/) applies to the data made available in this article, unless otherwise stated in a credit line to the data. 
Conclusions: Our data indicate that pre-existing infection of T. spiralis may suppress P. berghei parasitaemia and aggravate malaria-induced liver pathology through stimulating Gal-1 and Gal-3 expression, activating macrophages, neutrophils, and eosinophils, and promoting mediator release and cytokine production.

Keywords: Mice, P. berghei, T. spiralis, Co-infection, Liver pathology, Galectins, Macrophages, Neutrophils, Eosinophils

\section{Background}

The manifestations of severe malaria often present clinically as cerebral malaria, pulmonary edema, acute kidney injury, hypoglycaemia, lactic acidosis, anemia, and liver involvement [1]. Helminths and malaria are among the most prevalent infectious diseases in the world [2]. Malaria remains a serious public health issue in subSaharan region, while soil-transmitted helminths, such as Ascaris lumbricoides, Trichuris trichiura, and the hookworm species, infect more than a billion people worldwide [3], which have wide geographical overlap with malaria prevalent areas [4]. However, how concurrent infections of parasites affect the epidemiology and pathogenesis of each other remains controversial. It has been reported that the incidence rate of malaria attacks was significantly higher among Schistosoma mansoniinfected patients [5]. Mixed Plasmodium falciparum and $P$. vivax infections were found to be more frequent in A. lumbricoides-infected patients in Thailand, while infection with $A$. lumbricoides was associated with a dose-dependent effect on protection from cerebral malaria and acute renal failure [6]. Furthermore, a controlled randomized trial of anti-helminthic treatment showed a negative interaction between $A$. lumbricoides and $P$. falciparum malaria parasite density in Malagasy population (over 5 years-old), yielded to a higher malaria transmission [7]. However, in southern Ethiopia, T. trichiura infection was associated with increased malaria prevalence, while an increased worm burden of helminths as expressed by egg intensity was associated with increased malaria parasitaemia [4]. In addition, intensity of hookworm infection was associated with increased $P$. falciparum or $P$. vivax parasitemia [8]. In Uganda, $P$. falciparum and Taenia spp. co-infections constitute an important risk to anemia and malaria in the co-infected children [9]. It has been shown that heavy $A$. lumbricoides and T. trichiura infections were associated with high $P$. falciparum parasitaemia in a peri-urban community in Kwara State, Nigeria [10].

Trichinella spiralis can infect humans and more than 150 types of other animals via eating raw or undercooked pork, causing trichinellosis, a globalized zoonotic parasitic disease [11]. Cases of trichinellosis have been reported worldwide except for Antarctica [12, 13]. Trichinella spiralis infection in rodents has been used extensively to study the impact of inflammation on the small intestine
[14]. Trichinella spiralis infection can induce an increase of small intestine mucosal immune cells, and the intestinal mucosal immune system of infected mice was induced toward mixed Th1/Th2 phenotypes with the predominance of Th2 response at the early stage of infection [15]. Arrival of newborn larvae in muscle is coincident with an intestinal Th2 immune response that expels adult worms and induces prominent blood and tissue eosinophilia [16].

So far, the impact of helminths on liver immunopathology induced by malaria is not well-understood. PreT. spiralis-infected animal model has been used to study Th2 immune response on malaria by some researchers $[17,18]$. Therefore, in the present study we established a mouse model co-infected with the rodent blood-stage malaria parasite $P$. berghei of strain ANKA (PbANKA) and a pre-existing nematode $T$. spiralis to assess the influence of concurrent helminth parasite infection on the development of malarial hepatopathy. We found that co-infection with $T$. spiralis increased the immune response and exacerbated PbANKA malaria-induced liver immunopathology by altering a number of key immune factors.

\section{Methods}

\section{Mice, parasites, and infections}

Female, 6-8 weeks-old Kunming mice were purchased from the animal facility at Sun Yat-sen University in Guangzhou, China. Trichinella spiralis (pig strain) was maintained in our laboratory via serial passage in mice, and the larvae were recovered from muscles of mice at 60-90 days post-infection (pi). Standard procedures were used for isolation, collection, and inoculum [19]. PbANKA parasites were maintained at liquid nitrogen as a cryo-frozen stock of parasitized red blood cells (pRBCs) in our laboratory. The parasites were prepared through 3 cycles of passage of the pRBCs in mice. The experimental mice were each infected with $1 \times 10^{6}$ pRBCs by intraperitoneal (ip) injection. A total of 70 mice were used in this experiment. Mice were randomized into 4 groups; except for the uninfected control group consisting of 10 mice, the other 3 groups consisted of 20 mice per group. Trichinella spiralis infection was performed 9 days prior to PbANKA infection: (i) uninfected control group; (ii) T. spiralis-infected group: mice were mono-infected with $20 \mathrm{~T}$. spiralis larvae by oral gavage on day 0 that was assumed as the day of $T$. 
spiralis inoculation; (iii) PbANKA-infected group: mice were mono-infected with $1 \times 10^{6} \mathrm{PbANKA}$ in $0.1 \mathrm{ml}$ of PBS administered by ip injection; and (iv) co-infected group: mice were infected with $20 \mathrm{~T}$. spiralis and 9 days later challenged with $1 \times 10^{6} \mathrm{PbANKA}$. Mortality of each mouse was monitored daily, body weight was monitored from day 4 pi, and parasitemia was monitored daily by Giemsa-stained thin blood smears of tail blood. Erythrocyte counts were performed with a hematocytometer, and more than 1000 RBCs were counted under $1000 \times$ oil-immersion light microscopy to determine the percentage of pRBCs [20].

\section{Measurement of serum enzyme activities}

Mice were euthanized in a chamber by $\mathrm{CO}_{2}$ asphyxiation on day 22 after $T$. spiralis infection and/or on day 13 after PbANKA infection. The blood samples of mice were collected and centrifuged at $800 \times g$ for $10 \mathrm{~min}$. After centrifugation, the supernatant was collected and stored at $-20{ }^{\circ} \mathrm{C}$ until further use. Serum levels of aspartic aminotransferase (AST) and alanine aminotransferase (ALT) were measured by different assay kits (ALTL/ASTL; Roche, Mannheim, Germany) to determine the liver damage using an automatic biochemical analyzer (RocheHitachi cobas 8000 c702 Chemistry Autoanalyzer; Roche Diagnostics, Tokyo, Japan) according to the manufacturer's instructions.

\section{Determination of the liver and spleen indexes}

On day 22 after $T$. spiralis infection and/or on day 13 after PbANKA infection, the body weight of each mouse was measured before sacrificed, and the livers and spleens of mice were excised and weighed. Liver index $=$ liver weight $(\mathrm{g}) /$ body weight $(\mathrm{g}) \times 100 \%$, and spleen index $=$ spleen weight $(\mathrm{g}) /$ body weight $(\mathrm{g}) \times 100 \%$.

\section{Histopathological analyses}

Mice were euthanized by $\mathrm{CO}_{2}$ asphyxiation on day 22 after T. spiralis infection and/or on day 13 after PbANKA infection, and livers and spleens were harvested and immediately fixed in $10 \%$ buffered natural formaldehyde (Guangzhou Chemical Reagent Factory, Guangzhou, China) for over $48 \mathrm{~h}$. Four-micrometer-thick serial tissue sections of the organs from each mouse were stained with hematoxylin and eosin (H\&E) (Sigma-Aldrich, St. Louis, MO, USA) and imaged under light microscopy. To evaluate the hepatic histological alteration, a semiquantitative scoring system was used. The liver lesions were scored for 4 parameters as previously described, including (i) architecture loss, (ii) pRBCs in microvessels, (iii) pigment deposition, and (iv) portal tract inflammation [21, 22]. The liver damage score ranged from 0 to 3
( 0 , normal; 1 , mild; 2 , intermediate; and 3, most serious). Score 0 meant no histopathological change and score 12 referred to the most severe histopathological change. Scoring of each tissue sample represented the mean score of at least five separate microscopic fields at a magnification of $400 \times$.

\section{Immunohistochemistry}

After the liver and spleen tissue sections $(4 \mu \mathrm{m})$ were deparaffinized and rehydrated in distilled water, heatinduced antigen retrieval was performed in citrate buffer in an $800 \mathrm{~W}$ microwave oven for $30 \mathrm{~min}$. Sections were treated with $3 \%$ hydrogen peroxide in methanol for $10 \mathrm{~min}$ at room temperature to inactivate endogenous peroxidase, and then incubated in $10 \%$ bovine serum albumin in phosphate buffered solution (PBS, $\mathrm{pH}$ 7.4) for $60 \mathrm{~min}$ at room temperature to block non-specific binding. After washing with PBS, sections were incubated with the primary antibodies including CD68 (1:200, Bsm-33056m; Beijing Biosynthesis Biotechnology Co., Ltd., Beijing, China), neutrophil elastase (1:100, PB1114; Wuhan Boster Biological Engineering Co., Ltd., Wuhan, China), galectin (Gal)-1 (1:250, A00470; Wuhan Boster Biological Engineering Co., Ltd.), or Gal-3 (1:200, Bs0721r; Beijing Biosynthesis Biotechnology Co., Ltd.) at $37^{\circ} \mathrm{C}$ for $1 \mathrm{~h}$. Negative controls were performed without a primary antibody. Then, slides were incubated with secondary antibody (goat anti-mouse/rabbit IgG, PV-9001 kit; Beijing Zhongshan Golden Bridge Biotechnology Co., Ltd., Beijing, China). Antigens were visualized using a DAB kit (ZLI-9017; Beijing Zhongshan Golden Bridge Biotechnology Co., Ltd.). Four non-repeating immunohistochemical images of each sample were visualized and acquired using Olympus BX63 microscope image system (Olympus, Tokyo, Japan) under high power field (magnification of $400 \times$ ). The immunohistochemical signals (positive areas) were quantified using images captured with a digital camera system and analyzed by using Image-Pro Plus software (Image Z1 software, version 6.0; Media Cybernetics, Silver Spring, MD, USA).

\section{Isolation of murine peritoneal macrophages}

Following $T$. spiralis infection on day $22 \mathrm{pi}$ and/or PbANKA infection on day $13 \mathrm{pi}$, naive mice, T. spiralisinfected mice, PbANKA-infected mice, and T. spiralis + PbANKA-co-infected mice were ip injected with 2 $\mathrm{ml}$ of $3 \%$ thioglycollate broth (Sigma-Aldrich) once daily for 3 days. Animals were sacrificed and their peritoneal macrophages were harvested by peritoneal wash with 2 $\mathrm{ml}$ PBS containing $10 \%$ fetal calf serum (FBS), which was repeated 3 times. Peritoneal lavage fluid was centrifuged at $800 \times g$ at $4{ }^{\circ} \mathrm{C}$ for $5 \mathrm{~min}$, and the pelleted peritoneal 
macrophages were re-suspended in RPMI 1640 culture medium containing $10 \% \mathrm{FBS}$ and seeded at $5 \times 10^{5}$ cells/ well in 12-well plates (Corning, NY, USA) at $37^{\circ} \mathrm{C}$ in a $5 \%$ $\mathrm{CO}_{2}$ atmosphere. After $2 \mathrm{~h}$, the non-adherent cells were removed, and adherent cells were washed and collected. Samples were stored at $-80{ }^{\circ} \mathrm{C}$ until subjected to further mRNA analysis.

\section{Determination of mRNA expression by using quantitative real-time reverse transcription-polymerase chain reaction (qRT-PCR)}

Total RNA was extracted from about $100 \mathrm{mg}$ of liver and spleen tissues and peritoneal macrophages isolated from each mouse using an RNA extraction kit (TaKaRa, Shiga, Japan) as per the manufacturer's protocol. RNA amount was determined by measuring the ratio of absorbance at 260 and $280 \mathrm{~nm}$ using a NanoDrop ND-1000 spectrophotometer (NanoDrop Technologies, Wilmington, USA). First-strand cDNA was constructed from $1.0 \mu \mathrm{g}$ of total RNA with oligo(dT) as primers using a PrimeScript 1st Strand cDNA Synthesis Kit (TaKaRa). To determine tissue mRNA levels of Gal-1, Gal-3, cytokines (IFN $\gamma$, IL-1 $\beta$, IL-6, iNOS, and Ym1), C-C motif chemokine ligand 11 (CCL11), CCL24, eosinophil cationic protein (ECP), and eosinophil protein $\mathrm{X}$ (EPX), qRT-PCR measurements were performed using SYBR Green QPCR Master Mix (TaKaRa). Primers are listed in Table 1. Briefly, a total of $10 \mu \mathrm{l}$ reaction mixture contained $5.0 \mu \mathrm{l}$ of $\mathrm{SYBR}^{\circledR}$ Premix Ex TaqTM $(2 \times), 0.5 \mu \mathrm{l}$ of each primer $(10 \mathrm{pM})$, $3.0 \mu \mathrm{l}$ of $\mathrm{dH}_{2} \mathrm{O}$, and $1.0 \mu \mathrm{l}$ of cDNA $(0.2 \mu \mathrm{g} / \mu \mathrm{l})$. Amplification was pre-denaturized for $30 \mathrm{~s}$ at $95{ }^{\circ} \mathrm{C}$, followed by 43 cycles of $5 \mathrm{~s}$ at $95{ }^{\circ} \mathrm{C}$ and $20 \mathrm{~s}$ at $60{ }^{\circ} \mathrm{C}$ with a LightCycler $^{\circledR} 480$ instrument (Roche Diagnostics). The results are expressed as fold change compared with uninfected controls.

\section{Statistical analysis}

Statistics and graphing of numerical data were performed using GraphPad Prism7 software (GraphPad Software, La Jolla, CA, USA). Data are presented as means \pm standard deviation (SD) from at least 3 independent biological replicates. Differences in the survival curves were assessed with Log-rank (Mantel-Cox) test. One-way ANOVA with Bonferroni post-tests was used to compare differences among more than two different groups and two-way ANOVA with Bonferroni post-tests was used to compare differences of parasitemia and change in body weight among more than two different groups. Pearson's correlation coefficients were calculated to analyze correlations between the levels of galectins and cytokines. $P<0.05$ was considered to be statistically significant.

\section{Results}

Prior T. spiralis infection decreased parasitemia of PbANKA-infected mice without affecting overall survival To investigate whether prior T. spiralis infection alters the course of a subsequent malaria infection, we compared the changes in body weight, survival rate, and parasitemia of age-matched Kunming mice monoinfected with PbANKA or infected with T. spiralis and 9 days later challenged with PbANKA. Body weight were measured from day 4 following PbANKA infection, the results showed that $P b$ ANKA group and T. spiralis + PbANKA group had a similar tendency of body weight loss from days 7 to 14 pi. However, compared with $\mathrm{PbANKA-mono-infection,} \mathrm{significant} \mathrm{weight} \mathrm{loss}$ was observed on days 8,9 , and 10 post-co-infection (two-way ANOVA: $F_{(1,10)}=3.855, P=0.022, P=0.008$, and $P<0.001$, respectively) (Fig. 1a). Naive mice and T. spiralis-mono-infected mice all survived during the experiment. Mice of the PbANKA group died between

Table 1 Primer sequences of genes used for quantitative real-time reverse transcription-polymerase chain reaction assays

\begin{tabular}{llll}
\hline Gene & Forward primer $\left(5^{\prime}-3^{\prime}\right)$ & Reverse primer $\left(5^{\prime}\right.$-3 $\left.^{\prime}\right)$ & GenBank ID \\
\hline GAPDH & CAACTTTGGCATTGTGGAAGG & ACACATTGGGGGTAGGAACAC & NC_000072.6 \\
Gal-1 & GCCTACACTTCAATCCTCGCT & GTTCCCGGTGTTCGGTTCC & NM_008495 \\
Gal-3 & GGAGAGGGAATGATGTGCCT & TCCTGCTTCGTGTTACACACA & NM_001145953 \\
CCL11 & GAATCACCAACAACAGATGCAC & ATCCTGGACCCACTTCTTCTT & NC_000077.6 \\
CCL24 & ATTCTGTGACCATCCCCTCAT & TGTATGTGCCTCTGAACCCAC & NM_019577 \\
ECP & ATCCAAGTGGCTTGTGCAGTGAC & TAAGGTGTTCTCCTCCGACTGGTG & NC00080.6 \\
EPX & CTCACCCAACACGCTGAAG & TTTTCTGTGTGTGATTGTAGGCA & NM_007946 \\
IFNY & CCATCCTTTTGCCAGTTCCTC & NM-008337 \\
IL-1 13 & ATGAACGCTACACACTGCATC 6 & ATCTTTTGGGGTCCGTCAACT & NC_000071.6 \\
iNOS & GCAACTGTTCCTGAACTCAACT & CATTTCCACGATTTCCCAGA & NM_010927 \\
Ym1 & CCGGAGAGGAGACTTCACAG & GTGGACGGGTCGATGTCAC & NC_000069.6 \\
\hline
\end{tabular}




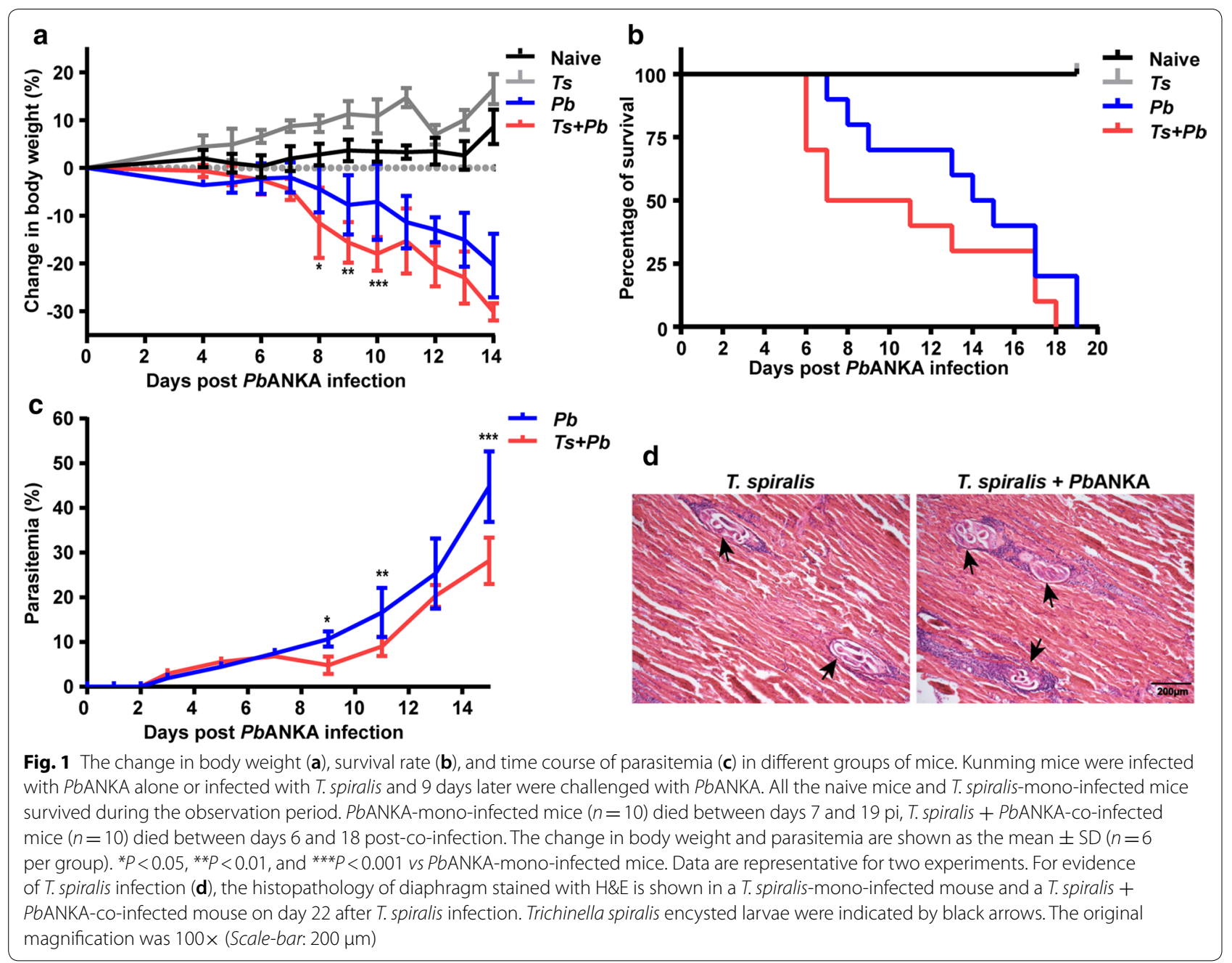

7 and 19 days pi, and approximately $50 \%$ of PbANKAmono-infected mice succumbed between 7 and 14 days pi; while mice of the $T$. spiralis + PbANKA group died between 6 and 18 days, and approximately $50 \%$ of T. spiralis + PbANKA-co-infected mice succumbed between 6 and 11 days post-co-infection. However, there were no significant differences in overall survival between the two groups (Log-rank test: $\chi^{2}=1.911, d f=1, P=0.167$ ) (Fig. 1b). Parasitemia was analyzed from day 3 after PbANKA infection. Although the levels of parasitemia were comparable for the PbANKA group and T. spiralis $+P b$ ANKA group from day 3 to 7 following PbANKA infection, the PbANKA group had significant higher percentages of infected erythrocytes than the T. spiralis + PbANKA group on days 9,11 , and 15 post- $P b$ ANKA infection (two-way ANOVA: $F_{(1,10)}=2.283, P=0.044$, $P=0.003$, and $P<0.001$, respectively) (Fig. 1c). For evidence of $T$. spiralis infection, on day 22 after infection with 20 muscle larvae of $T$. spiralis, the diaphragm tissues of both T. spiralis-mono-infected and T. spiralis + PbANKA-co-infected mice were examined. Mice of both groups presented encysting larvae of $T$. spiralis in the skeletal muscles, which were surrounded by various inflammatory cells (Fig. 1d). Overall, the results demonstrated that prior T. spiralis infection decreases parasitemia levels but does not affect the survival outcome in a subsequent $P b A N K A$ infection.

Prior T. spiralis infection increased liver and spleen indexes and liver pathology of $P b A N K A$-infected mice

On day 22 after T. spiralis infection and/or on day 13 after PbANKA infection, the size of livers and spleens were notably different among the groups, hepatosplenomegaly was more marked in T. spiralis + PbANKA-coinfected mice compared with either infection separately (Fig. 2a). Liver and spleen indexes of different groups were determined by fixed calculation formula. Compared with naive mice or mice mono-infected with $T$. 
spiralis, there were significantly increased liver and spleen indexes in both PbANKA-mono-infected mice and $T$. spiralis + PbANKA-co-infected mice (one-way ANOVA: $F_{(3,20)}=105.2$ and $F_{(3,20)}=103.8$, respectively, $P<0.0001)$; whereas significantly increased liver and spleen indexes were found in co-infected mice com-

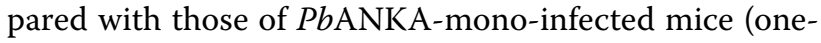
way ANOVA: $F_{(3,20)}=105.2, P=0.04$ and $F_{(3,20)}=103.8$, $P=0.002$, respectively) (Fig. $2 \mathrm{~b}$ ), suggesting that pre$T$. spiralis infection regulates the immune response of the immune organs (such as liver and spleen) to Plasmodium parasite infection. We further investigated whether prior $T$. spiralis infection affects liver function of PbANKA-infected mice. Compared with naive mice or
T. spiralis-mono-infected mice, there were significantly increased serum ALT in PbANKA-mono-infected mice (one-way ANOVA: $F_{(3,20)}=4.333, P=0.022$ ), and significantly increased serum AST in both PbANKA-monoinfected and T. spiralis + PbANKA-co-infected mice (one-way ANOVA: $F_{(3,20)}=37.8, P<0.0001$ ) (Fig. $2 \mathrm{c}$ ). However, there were no significant differences in serum ALT and AST levels between the PbANKA group and the T. spiralis + PbANKA group, suggesting prior T. spiralis infection had no protective effect on liver function damage induced by malarial infection.

The livers of different groups of mice were examined histologically. On day 22 after T. spiralis infection and/ or on day 13 after PbANKA infection, the liver tissues

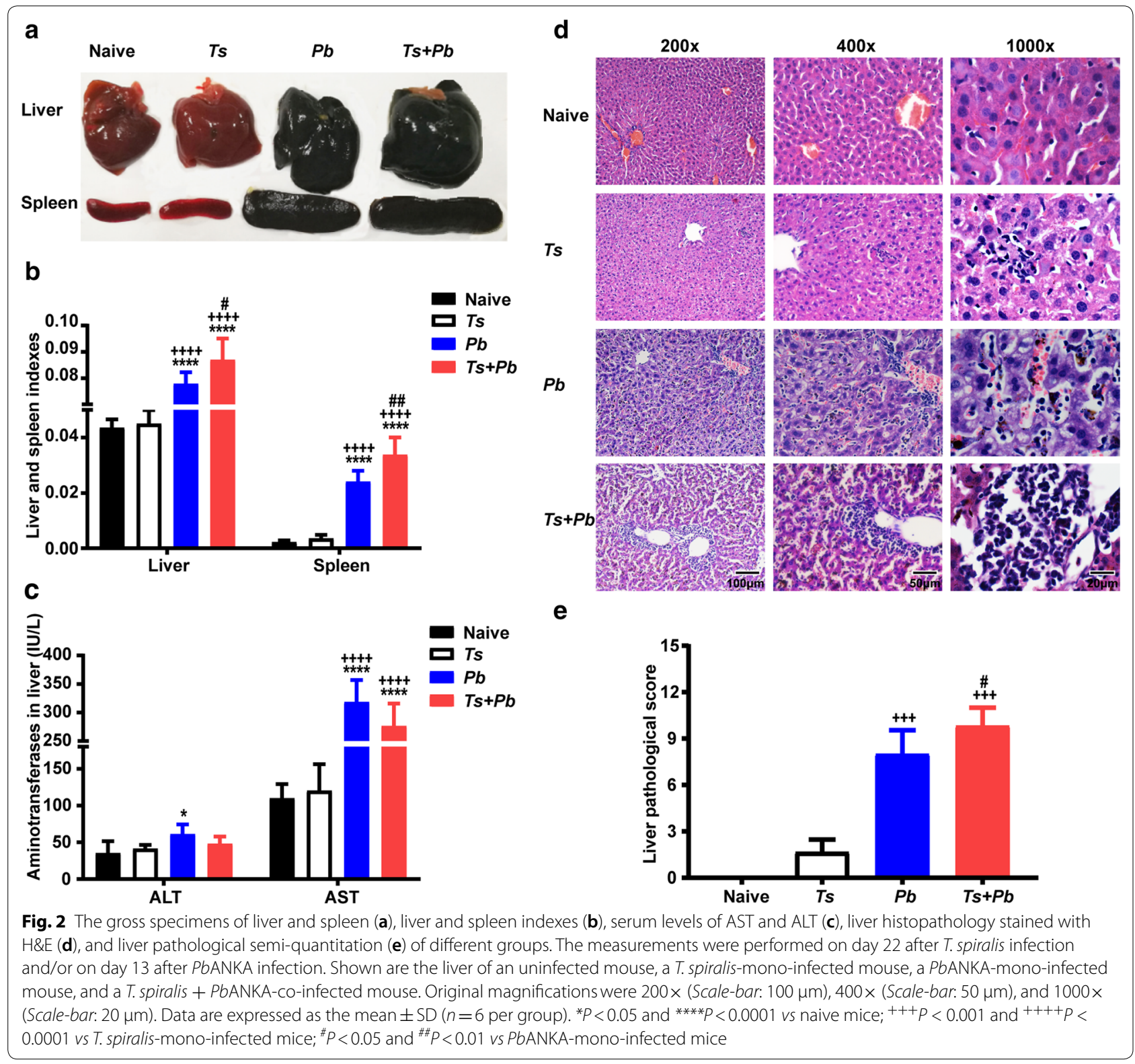


from naive mice showed no structural abnormalities. Acute small foci of inflammation were observed in the liver of mice with $T$. spiralis-mono-infection. However, the livers of both PbANKA and T. spiralis + PbANKA groups showed typical features of Plasmodium infection characterized by tissue edema, small necrotic foci, and malaria pigment deposition. Compared with PbANKA-mono-infected mice, there was more severe liver damage in co-infected mice, characterized by more malaria pigment deposition and inflammatory infiltration on day 13 post-co-infection (Fig. 2d). To evaluate liver damage after PbANKA infection, semiquantitative liver inflammation scores based on liver pathological changes showed that compared with $T$. spiralis-mono-infected mice, there were significantly increased pathological scores in both the PbANKA group and the T. spiralis + PbANKA group (one-way ANOVA: $\left.F_{(2,15)}=74.55, P<0.001\right)$; however, compared with $\mathrm{PbANKA-mono-infected} \mathrm{mice,} \mathrm{there} \mathrm{was} \mathrm{a} \mathrm{sig-}$ nificantly increased pathological score in the liver of co-infected mice (one-way ANOVA: $F_{(2,15)}=74.55$, $P=0.049$ ) (Fig. 2e), suggesting that prior T. spiralis infection resulted in accelerated liver pathology with a subsequent PbANKA infection.

\section{Prior T. spiralis infection increased the expression of Gal-1} and Gal-3 in the liver and spleen of PbANKA-infected mice Compared with naive mice or T. spiralis-mono-infected mice, increased immunohistochemical expression of Gal-1 (Fig. 3a) and Gal-3 (Fig. 3b) were observed in the liver and spleen sections of both PbANKA-monoinfected and T. spiralis + PbANKA-co-infected mice. Semi-quantitative immunohistochemical analysis showed that compared with PbANKA-mono-infected mice, there were significantly increased expression levels of Gal-1 and Gal-3 in the liver (one-way ANOVA: $F_{(3,20)}=86.93$ and $F_{(3,20)}=33.65$, respectively, $\left.P<0.001\right)$ and increased Gal-3 expression level in the spleen (one-way ANOVA: $F_{(3,20)}=8.867, P=0.002$ ) of co-infected mice (Fig. 3c, d). The data provide evidence that Gal-1 and Gal-3 may be involved in the pathogenic basis of malaria.

\section{Prior T. spiralis infection increased $\mathrm{CD} 68^{+}$macrophage and neutrophil elastase expression levels in the liver and spleen of PbANKA-infected mice}

Neutrophil elastase is a cytotoxic serine protease, which is stored in the azurophil granules of neutrophil granulocytes and is released by activated neutrophils. CD68 ${ }^{+}$ macrophages and elastase-positive neutrophils were detected in the liver and spleen sections of both the PbANKA group and the $T$. spiralis + PbANKA group on day 13 post-PbANKA infection. Immunohistochemical staining showed that there were few $\mathrm{CD}^{+} 8^{+}$macrophages and no elastase-positive neutrophils observed in the liver and spleen of uninfected controls or the liver of $T$. spiralis-mono-infected mice. However, a large number of $\mathrm{CD}^{+} 8^{+}$macrophages (Fig. 4a) and elastase-positive neutrophils (Fig. 4b) were observed in the spleen of T. spiralis-mono-infected mice, and the liver and spleen of both PbANKA-mono-infected and T. spiralis + PbANKA-coinfected mice. Semi-quantitative analysis showed that compared with naive mice, there were significantly increased expression levels of $\mathrm{CD}^{+} 8^{+}$macrophage and neutrophil elastase in the liver (one-way ANOVA: $F_{(3,20)}=167.8$, $P<0.001$ and $F_{(3,20)}=41.41 P=0.003$, respectively) and spleen (one-way ANOVA: $F_{(3,20)}=55.2, P=0.002$ and $F_{(3,}$ 20) $=26.33, P=0.032$, respectively) of PbANKA-monoinfected mice, and significantly increased expression levels of $\mathrm{CD} 8^{+}$macrophage and neutrophil elastase in the liver (one-way ANOVA: $F_{(3,20)}=167.8$ and $F_{(3,20)}=41.41$, respectively, $P<0.001$ ) and spleen (one-way ANOVA: $F_{(3,}$ ${ }_{20)}=55.2$ and $F_{(3,20)}=26.33$, respectively, $\left.P<0.001\right)$ of $T$. spiralis + PbANKA-co-infected mice. Compared with $P b A N K A-m o n o-i n f e c t e d$ mice, there were significantly increased expression levels of $\mathrm{CD}^{+} 8^{+}$macrophage and neutrophil elastase in the liver (one-way ANOVA: $F_{(3}$, ${ }_{20)}=167.8$ and $F_{(3,20)}=41.41$, respectively, $\left.P<0.001\right)$ and spleen (one-way ANOVA: $F_{(3,20)}=55.2, P<0.001$ and $F_{(3 \text {, }}$ ${ }_{20)}=26.33, P=0.002$, respectively) of co-infected mice (Fig. 4c, d).

\section{Prior T. spiralis infection showed more eosinophils in the liver and increased levels of ECP and EPX in the spleen of PbANKA-infected mice}

By H\&E staining, no eosinophils were observed in the liver of naive mice. However, on day 22 after T. spiralis infection and/or on day 13 after PbANKA infection, more eosinophils were observed in the livers of the PbANKA group, and even more eosinophils were observed in the livers of the T. spiralis group and the T. spiralis + PbANKA group (Fig. $5 \mathrm{a}$ ). To determine whether the difference in liver damage was reflected in the activation status of eosinophils, liver and spleen from the $P b$ NAKA group or the $T$. spiralis $+P b$ ANKA group were analyzed for the expression of eotaxin (CCL-11 and CCL-24), ECP, and EPX. Compared with naive mice, there was significantly increased CCL11 levels in the liver (one-way ANOVA: $F_{(3,20)}=9.059$, $P=0.009$ ) and spleen (one-way ANOVA: $F_{(3,20)}=32.24$, $P<0.001)$ of PbANKA-mono-infected mice, and significantly increased levels of CCL11 and CCL24 in the spleen (one-way ANOVA: $F_{(3,20)}=32.24, P<0.001$ and $F_{(3,20)}=3.044, P=0.045$, respectively) of $T$. spiralis + PbANKA-co-infected mice. Compared with 


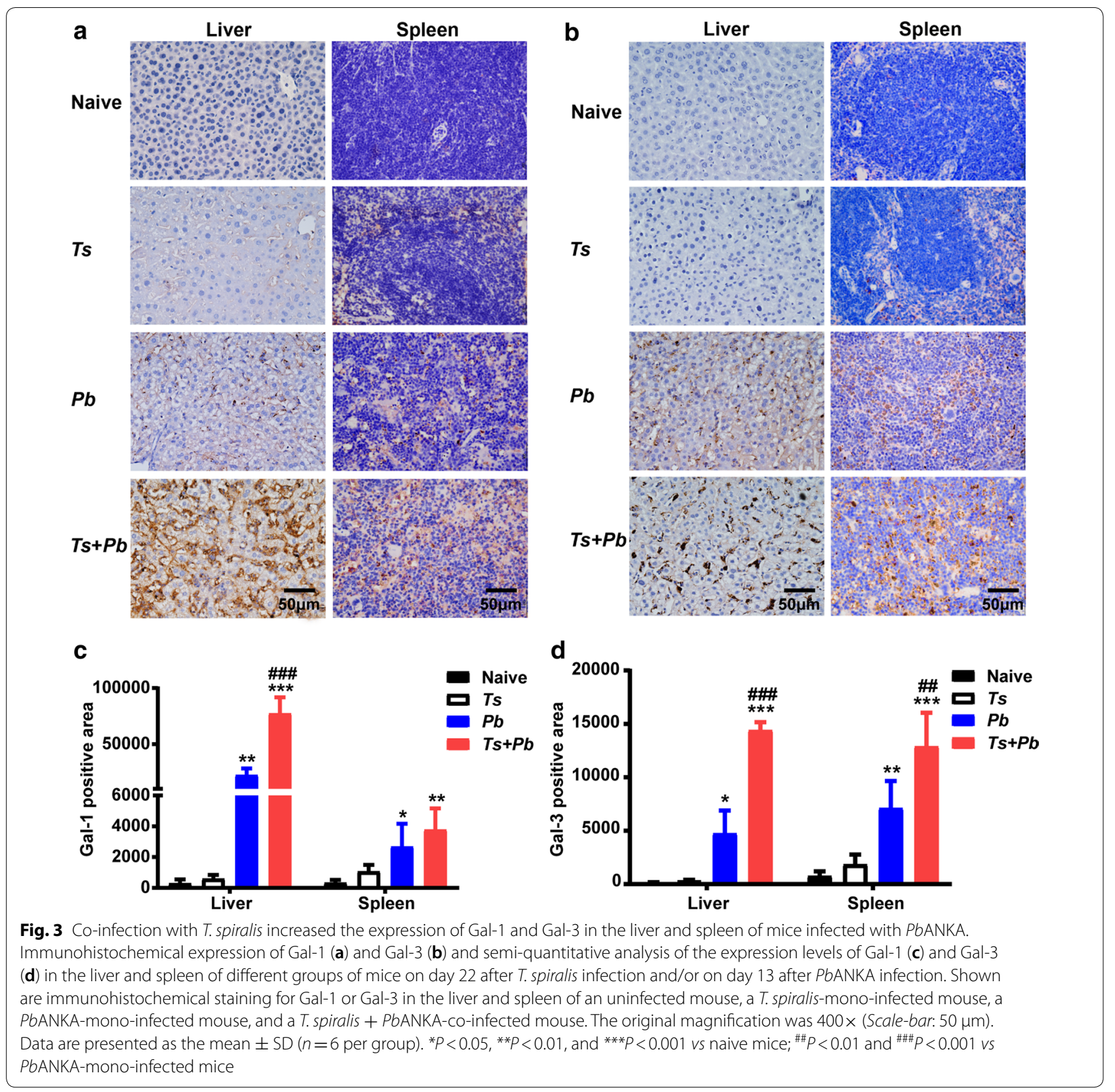

naive mice, there were significantly increased ECP levels in the livers (one-way ANOVA: $F_{(3,20)}=49.86$, $P<0.001, P<0.001$, and $P=0.005$, respectively) of $T$. spiralis-mono-infected, PbANKA-mono-infected, and co-infected mice, and in the spleen (one-way ANOVA: $\left.F_{(3,20)}=23.51, P<0.001\right)$ of $T$. spiralis-mono-infected mice, significantly increased EPX level in the liver and spleen of $T$. spiralis-mono-infected mice (one-way ANOVA: $F_{(3,20)}=11.34$ and $F_{(3,20)}=18.43$, respectively, $P<0.001)$, and significantly increased EPX level in the liver (one-way ANOVA: $F_{(3,20)}==11.34, P=0.012$ ) and spleen (one-way ANOVA: $F_{(3,20)}=18.43, P<0.001$ ) of co-infected mice. Compared with PbANKA-monoinfected mice, there were significantly increased levels of ECP (one-way ANOVA: $F_{(3,20)}=23.51, P=0.023$ ) and EPX (one-way ANOVA: $F_{(3,20)}=18.43, P<0.001$ ) in the spleen of co-infected mice (Fig. 5b). The data indicate that prior $T$. spiralis infection does significantly increase eosinophil activation, which may have 


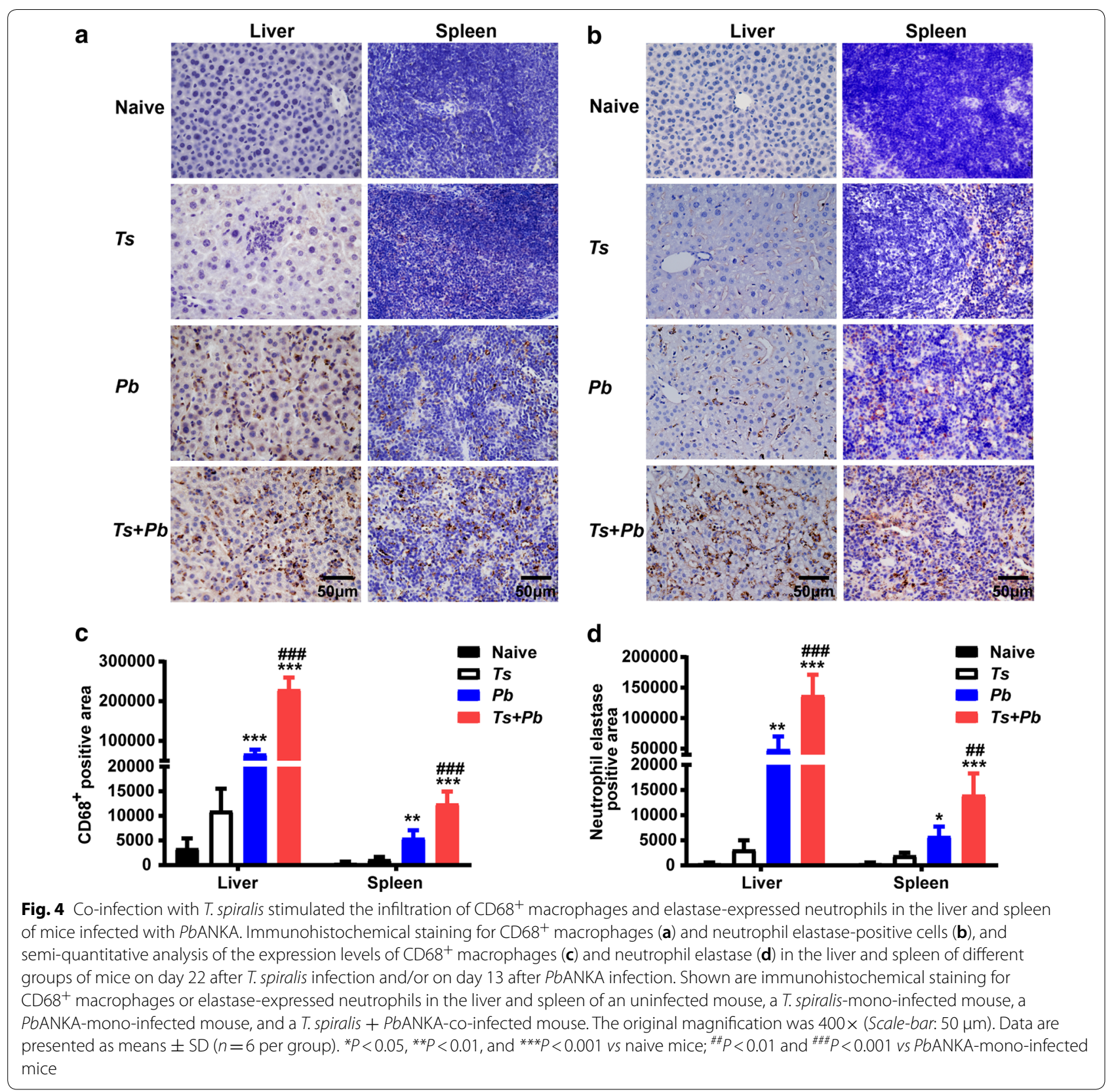

an effect on liver pathology of mice with a subsequent PbANKA infection.

Prior T. spiralis infection promoted the expression of Gal-1, Gal-3, IFNY, IL-6, IL-1 $\beta$, iNOS, and Ym1 in the liver, spleen, or peritoneal macrophage of $P b A N K A$-infected mice To determine the immune responses of the livers, spleens, and macrophages of different groups of mice, the mRNA expression of Gal-1, Gal-3, IFN $\gamma$, IL-1 $\beta$ (M1 marker), IL-6, iNOS (M1 marker), and Ym1 (M2 marker) in the peritoneal macrophage from different groups were measured on day 22 after T. spiralis infection and/or on day 13 after PbANKA infection. Compared with $P b A N K A-m o n o-i n f e c t e d$ mice, there were significantly increased expression levels of Gal-1 (oneway ANOVA: $F_{(3,20)}=48, P=0.004$ ), Gal-3 (one-way 


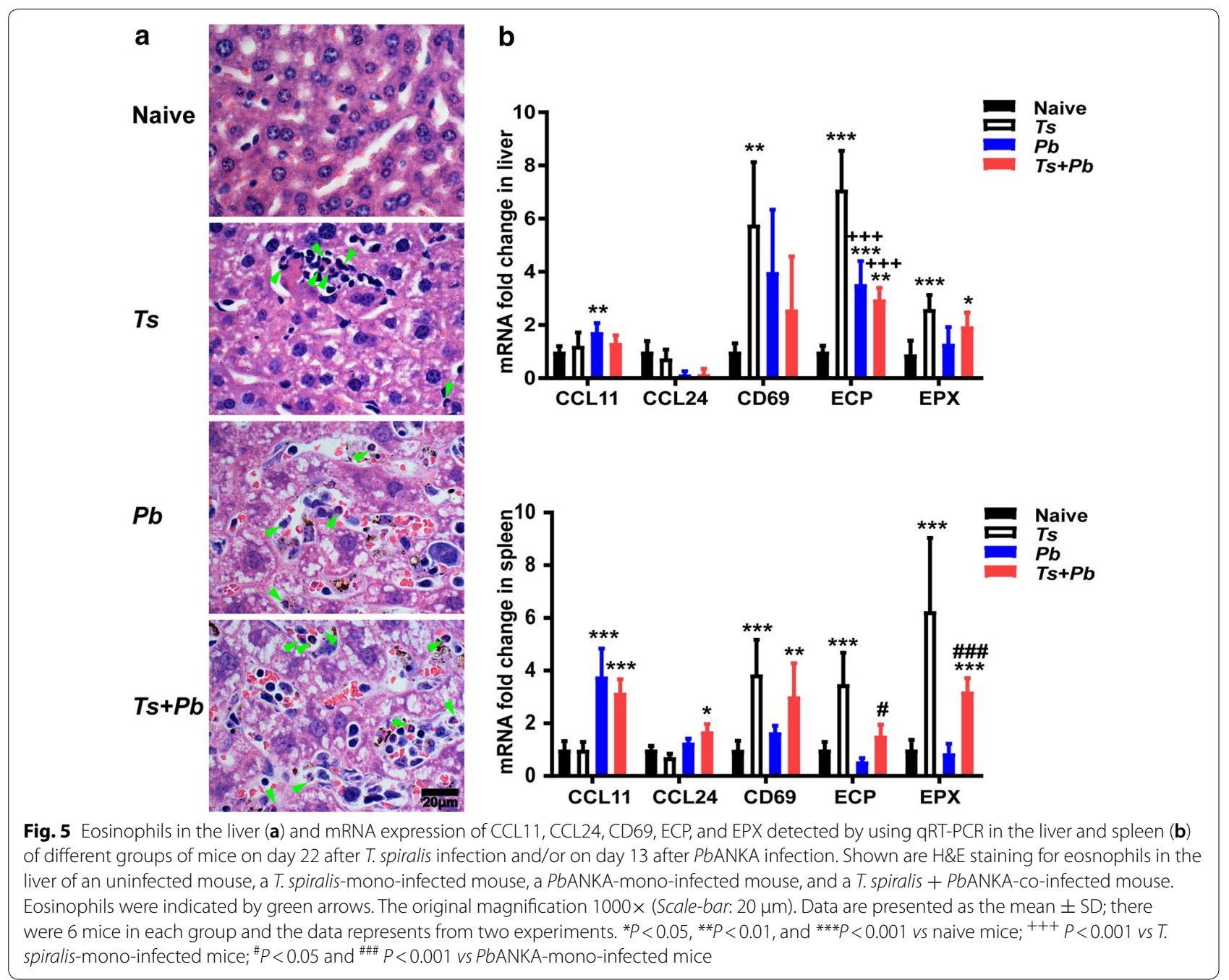

ANOVA: $\quad F_{(3,20)}=36.64, \quad P=0.006$ ), IFN $\gamma \quad$ (one-way ANOVA: $\quad F_{(3,20)}=13.06, \quad P=0.016$ ), IL-6 (one-way ANOVA: $F_{(3,20)}=45.25, P<0.001$ ), and iNOS (one-way ANOVA: $\left.F_{(3,20)}=28.49, P<0.001\right)$ in the liver; significantly increased expression levels of Gal-1 (one-way ANOVA: $\quad F_{(3,20)}=29.87, P=0.015$ ), IFN $\gamma$ (one-way ANOVA: $F_{(3,20)}=31.65, \quad P=0.005$ ), IL-1 $\beta$ (one-way ANOVA: $F_{(3,20)}==16.43, P=0.004$ ), IL-6 (one-way ANOVA: $\quad F_{(3,20)}=42.15, \quad P=0.005$ ), iNOS (one-way ANOVA: $F_{(3,20)}=105.4, P=0.009$ ), and $\mathrm{Ym} 1$ (one-way ANOVA: $\left.F_{(3,20)}=16.19, P=0.015\right)$ in the spleens, and significantly increased expression levels of Gal-1 (oneway ANOVA: $F_{(3,20)}=21.96, P=0.002$ ), Gal-3 (oneway ANOVA: $F_{(3,20)}=16.66, P=0.004$ ), IL-1 $\beta$ (one-way ANOVA: $F_{(3,20)}=46.2, P<0.001$ ), IL-6 (one-way ANOVA: $F_{(3,20)}=56.11, P=0.024$ ), and iNOS (one-way ANOVA: $\left.F_{(3,20)}=134.6, P<0.001\right)$ in the peritoneal macrophages of
T. spiralis + PbANKA-co-infected mice (Fig. 6a). The data indicate that prior T. spiralis infection markedly increases Gal-1, Gal-3, and cytokine responses in the livers, spleens, and peritoneal macrophages of mice induced by malaria infection.

\section{Correlations between Gal-1/Gal-3 and IFN $\gamma$, IL-1 $\beta$, IL-6, iNOS, or Ym1 in the liver, spleen, and peritoneal macrophages of different groups}

The correlations between mRNA levels of Gal-1/Gal-3 and IFN $\gamma$, IL-1 $1 \beta$, or Ym1 in the liver, spleen, and peritoneal macrophages of different groups of mice were analyzed, only significant correlations are provided here. Significant correlations were found between Gal-3 and IL-1 $\beta$ (Pearson's correlation: $r=0.799, P=0.0163$ ) in the peritoneal macrophages from $P b A N K A-m o n o-i n f e c t e d$ mice on day 13 after infection, between Gal-3 and IFNY (Pearson's correlation: $r=0.8388, P=0.0103$ ) in the spleen 


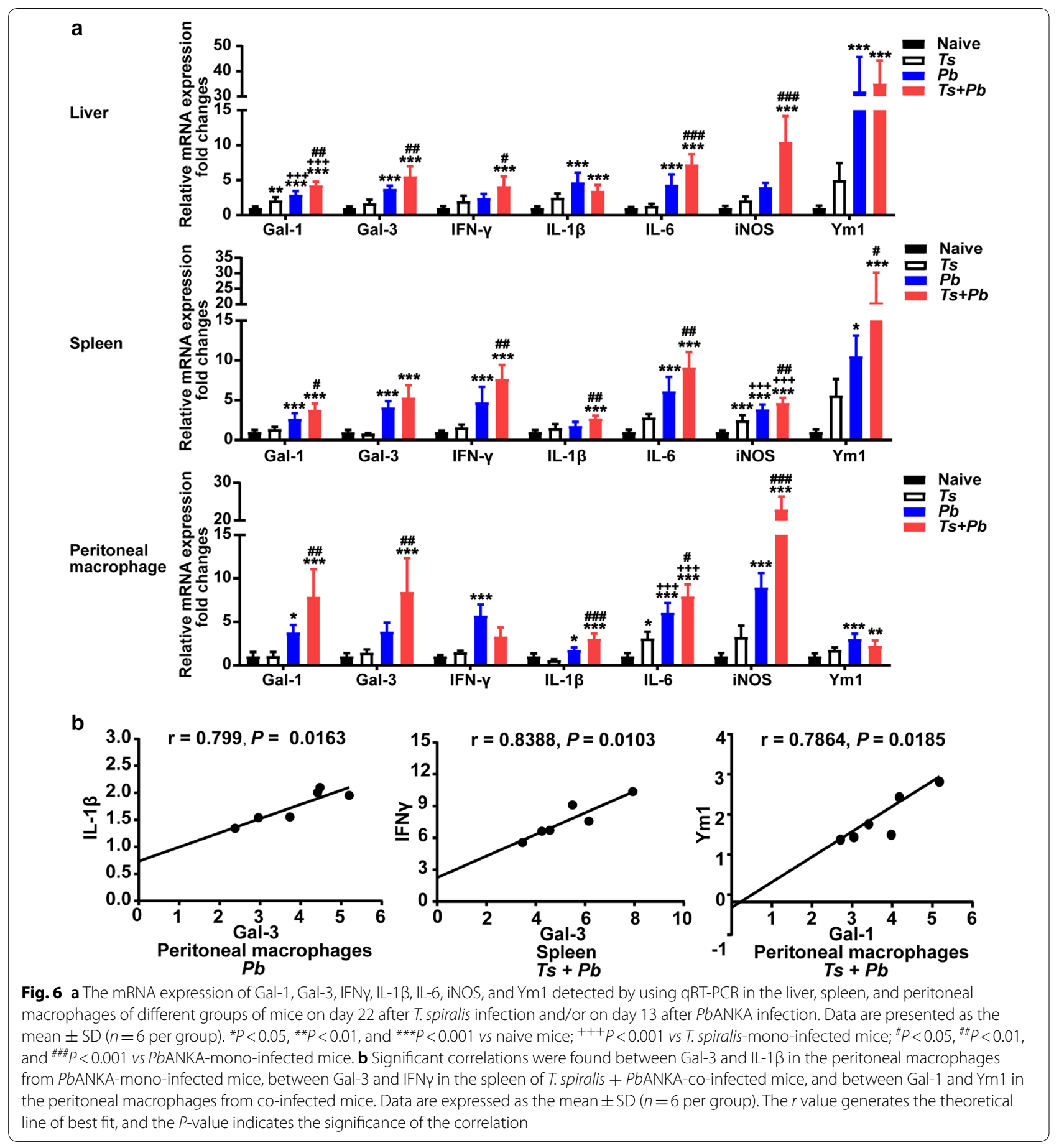

of T. spiralis + PbANKA-co-infected mice on day 13 after co-infection, and between Gal-1 and Ym1 (Pearson's correlation: $r=0.7864, P=0.0185)$ in the peritoneal macrophages from co-infected mice on day 13 after coinfection (Fig. 6b).

\section{Discussion}

Malaria caused by Plasmodium spp. is a major health problem in many countries in the tropical and subtropical regions of the world. Hepatocellular dysfunction is recognized as one of the clinical features in severe malaria [23]. However, the mechanism of hepatic injury during malaria is still unknown. Helminths exert 
strong immunomodulatory effects in their hosts [24]. In endemic regions, it is not uncommon for patients to be co-infected with helminths and malaria. A number of studies from different continents have shown complex interactions between different gastrointestinal nematodes (such as A. lumbricoides, T. trichiura, and hookworm) and malaria, with different immunomodulatory properties or conflicting consequences [6-8, 25]. Trichinella spiralis infection has been identified to have strong immunomodulatory effects [26]. Mice challenged with Listeria monocytogenes at 7 or 21 days after T. spiralis infection showed a higher mean lethal dose and longer survival time than control mice [27]. Infection with $T$. spiralis activates macrophages and mediators released by activated macrophages participate in the non-specific immunity [18]. So far, the effect of prior T. spiralis infection on $P$. berghei-induced liver disease has not been reported. To test the hypothesis that a pre-existing Th2 environment alters the general course of a subsequent malaria infection, in the present study, we compared the physiological and immunopathological responses of agematched Kunming mice mono-infected with PbANKA or infected with T. spiralis and nine days later challenged with PbANKA. Our data demonstrated that $T$. spiralis + PbANKA-co-infection results in statistically significant weight loss in only three days but markedly decreased parasitemia; however, the overall survival durations had no difference compared with PbANKA-mono-infected mice. It has been reported that children even with a light $S$. haematobium infection presented lower $P$. falciparum parasite densities than children not infected with S. haematobium [28]. However, mice co-infected with $S$. mansoni and P. chabaudi AS develop increased malaria parasitemia [29].

It has been reported that hepatosplenomegaly is more frequent in severe malaria [30]. Young children during malaria attacks have lymphocyte infiltrations in hepatic sinusoids, hyperplasia of Kupffer cells, and deposits of malaria pigment [31]. The reticular endothelial system of the liver is able to eliminate parasite-derived hemozoin and Plasmodium-infected erythrocytes through phagocytosis [32]. Exacerbated hepatosplenomegaly has been shown among Kenyan school children, living in a malaria and schistosomiasis co-transmission area [33, 34]. Hepatosplenomegaly caused by chronic exposure to malaria is clearly associated with increased circulating levels of proinflammatory mediators amongst S. mansoni-infected children [34]. Animal experiments also demonstrated that hepatosplenomegaly in BALB/c mice was much higher in the context of schistosome-malaria co-infection compared with infection of either P. yoelii or S. mansoni alone [35]. ALT and AST are primarily expressed in liver cells, when the physiological and biochemical function and the integrity of hepatocytes are damaged, resulting in elevated serum AST and ALT levels [36]. In the present study, compared with PbANKA-mono-infected mice, more severe hepatosplenomegaly and more overt hepatic pathology with increased hepatic inflammatory infiltrates of $\mathrm{CD} 8^{+}$macrophages, elastase-positive neutrophils, and eosinophils were measured, but similar serum levels of liver function enzymes ALT and AST were examined in the co-infected mice on day 13 post-PbANKA infection.

Peripheral blood mononuclear macrophages can be generally divided into classically activated macrophages (M1) and alternatively activated macrophages (M2). M1 macrophages, activated by Th1 cytokines of the infected host, upregulate IL-12 and iNOS expressions. In contrast, M2 macrophages, driven by the Th2 cytokines IL-4 and IL-13, express high levels of arginase-1, Ym1, mannose receptor, and resistance-like molecule- $\alpha$ [37, 38]. Tissue resident macrophages play key roles in infection with either PbANKA [20] or T. spiralis [39]. Prior Nippostrongylus brasiliensis infection has a lasting effect on lung macrophages, where PbANKA-induced M1-like response was reduced by previous M2 polarization [40]. Kupffer cell phagocytic activity in liver is markedly increased in rats with a high parasitemic load of malarial P. berghei infection [41], and electron microscopy revealed that Kupffer cells fill with damaged RBCs, pRBCs, and haemoglobin degradation pigment [42]. In the present study, mice were infected with $T$. spiralis and nine days later were challenged with PbANKA, and the functional characteristics of macrophages induced by prior $T$. spiralis infection in the regulation of immune reaction and inflammation in co-infected mice were examined. The ability of $T$. spiralis infection to modulate macrophage activation was determined by detecting the production of effector molecules including gelactins, iNOS, Ym1, and cytokines. Interestingly, $\mathrm{CD}^{+} 8^{+}$macrophage expression levels were significantly increased in the liver and spleen of co-infected mice compared with PbANKA-mono-infected mice. There were significantly increased expression levels of Gal-1, Gal-3, IL-1 $\beta$, IL-6, and iNOS in the peritoneal macrophage isolated from coinfected mice than that isolated from $P b A N K A-m o n o-$ infected mice. It is worth noting that M1 macrophage markers were increased in the liver, spleen, or peritoneal macrophages of co-infected mice, suggesting that the immune system of co-infected mice developed a M1 polarization of macrophages with an emerging predominance of IL-1 $\beta$, IL- 6 , and iNOS response, which may contribute to the liver damage of co-infected mice. However, it has been reported that increased Gal-3 expression and secretion is a feature of M2 macrophage activation [43]. 
Neutrophil activation and circulating neutrophil extracellular traps are elevated in human malaria, which may contribute to pathogenesis of severe falciparum malaria [44]. Neutrophil activation is also associated with pathogenesis of experimental cerebral malaria and acute lung injury/acute respiratory distress syndrome in murine models $[45,46]$. An in vitro study demonstrated that neutrophils can kill $P$. falciparum parasites [47]. In addition, after transferring neutrophils obtained from the spleens of $P b A N K A$-infected adult rats to young rats, they exerted a significant dose-dependent anti-parasite effect, demonstrating the role of neutrophils and their effector proteins in the control of early phase blood Plasmodium parasite growth [48]. The immune response of long-lasting infection of muscles with Trichinella is mainly characterized by a Th2 phenotype [49]. The present study showed that compared with PbANKA-mono-infected mice, there were significantly increased neutrophil elastase expression levels in the liver and spleen of $T$. spiralis + PbANKA-co-infected mice, indicating neutrophils may have detrimental effects on either controlling malaria parasites or aggravating liver pathology during the co-infection.

It has been reported that an eosinophilia is induced in P. falciparum malaria and the concentrations of eosinophil-derived granule proteins (ECP and EPX) are higher in patients with cerebral malaria than in uncomplicated cases or cases of severe malarial anemia, indicating eosinophil granule proteins are important in both the control of malaria infection and pathogenesis of severe malaria [50]. An in vitro study showed that the secreted products from eosinophils can kill $P$. falciparum parasites [51], but eosinophils may also contribute to pathology by release of granule proteins such as ECP and EPX/ eosinophil-derived neurotoxin [52]. Eotaxin is a member of the CC chemokine family, including three subfamilies, e.g. CCL11/eotaxin-1 [53], CCL24/eotaxin-2 [54], and CCL26/eotaxin-3 [55]. Chemokines CCL11 and CCL24 produced in the liver following halothane treatment play a role in attracting eosinophils to the liver [56]. Trichinella spiralis induces a pronounced eosinophilia that coincides with establishment of larval stages in skeletal muscles [57], and eosinophils served to limit the number of newborn larvae of $T$. spiralis that migrated in the tissues and colonized the skeletal muscles [58]. Eosinophils, as crucial to immune regulation, support the survival of muscle larvae of T. spiralis [57]; meanwhile eosinophils can be toxic for host tissues [59]. In the present study, we observed that eosinophils were increased in the liver of $T$. spiralis + PbANKA-co-infected mice on day 13 after coinfection. Measured via qRT-PCR, the mRNA levels of ECP and EPX were significantly increased in the spleen of co-infected mice compared to PbANKA-mono-infected mice, indicating that eosinophil and eosinophil granule proteins may play an important role in the pathogenisis of T. spiralis-malaria concurrent infection.

Galectins are a family of lectins, which contain conserved carbohydrate-recognition domains for $\beta$-galactosides [60]. Host galectins have been shown to modulate the effector function of mast cells, neutrophils, and eosinophils [61]. Gal-1 modulates protective $\mathrm{CD} 4^{+} \mathrm{T}$ cell responses during acute hepatic Leishmania donovani infection by limiting IFN $\gamma$-producing $\mathrm{CD}^{+}{ }^{+} \mathrm{T}$ cell numbers [62]. Gal-1 can induce human neutrophil migration [63], reduce eosinophil recruitment to the airways in a mouse model [64], and modulate macrophage polarization and protein secretion in an in vitro study [65]. Soluble Gal-1 enhances T cell IL-10 production and decreases cytotoxic $\mathrm{T}$ cell survival, reduces macrophage responses to IFNY [66], and promotes an M2-like macrophage phenotype [67]. Gal-1 immunoregulatory function is shown to contribute to enhanced parasite control, survival, and Th1 effector function in Gal-1-deficient mice infected with Trypanosoma cruzi [68]. Gal-3 is abundantly expressed and secreted by macrophages [69]. It has been demonstrated that alternative macrophage activation induced by extracellular Gal-3 via IL-4/IL-13 is repressed in Gal-3-deficient mice [43]. Gal-3-deficient mice are partially protected against experimental cerebral malaria caused by PbANKA infection but developed higher peripheral parasitemia [70]. Studies also demonstrated that Gal-3 facilitates neutrophil activation [71, 72], and Gal-3 bound to the cell surface of eosinophils is essential for eosinophil trafficking under flow and migration [73]. Gal-3 regulates the capacity of dendritic cells to support NKT-cell-mediated liver injury, playing an important pro-inflammatory role in acute liver injury [74]. The immunoregulatory role of Gal-1 and Gal-3 is relevant for the induction of the host response during liver invasion by Entamoeba histolytica [75]. So far there have been no reports regarding the role of Gal-1 in malaria. Our findings demonstrated that co-infection resulted in higher levels of mRNA levels of Gal-1, Gal-3, IFN $\gamma$, IL-6, and iNOS in the liver of co-infected mice compared with PbANKA-mono-infection. Since co-infected mice displayed inflammatory infiltrates and severe pathology of the liver, which indicates that Gal-1 and Gal-3 may have effects on both parasitemia control and hepatic injury. In addition, compared with PbANKA-mono-infection, co-infection resulted in elevated Gal-1, Gal-3, M1 markers (IL- $1 \beta$ and iNOS), and IL- 6 in the peritoneal macrophages, which may be associated with enhanced liver immunopathology during the co-infection. It has been reported that inflammatory cytokines IFNY and IL-6, which have elevated expression locally in the brain and systemically in the plasma, may represent surrogate 
markers for neutrophil-mediated response [76]. Gal-1 and Gal-3 are important mediators of inflammation, and both have the capacity to induce a respiratory burst in neutrophils. The reactive oxygen species produced may be destructive to the invading microorganisms as well as to the surrounding host tissue, indicating the possible role of galectins not only in defense toward infection but also in inflammatory-induced tissue destruction [77]. Notably, in the present study, significant correlations were found between Gal-3 and IL-1 $\beta$ in the peritoneal macrophages from PbANKA-mono-infected mice and between Gal-1 and Ym1 in the peritoneal macrophages and between Gal-3 and IFN $\gamma$ in the spleen of T. spiralis $+P b$ ANKA-co-infected mice. It has been reported that Gal-3 as an amplifier of IL- $1 \beta$ responses exacerbates the response to IL-1 $\beta$ by stimulating the secretion of inflammatory cytokines [78]. Our data suggest that Gal-1 and/ or Gal-3 play a regulatory role during malaria infection or during the co-infection with T. spiralis.

\section{Conclusions}

This study demonstrated that PbANKA infection is associated with an increased immune responsiveness that is affected by prior $T$. spiralis infection. Concurrent nematode infection strongly modulates multiple aspects of host immunity to blood-stage malaria, including immune cell function, cytokine production, and galectin responses. Consequently, the development of immunity to malaria is increased, leading to significantly decreased malaria parasitemia, exacerbated malaria-induced liver immunopathology, accompanied with elevated expression levels of Gal-1 and Gal-3, increased activation of macrophages, neutrophils, and eosinophils and increased production of their mediators in co-infected mice. Furthermore, our data demonstrated that a pre-existing helminth infection aggravates the secondary malaria infection, thus the control of helminth infection in a malaria epidemic area is of critical importance. Further study is needed to clarify the exact role of Gal-1 and Gal-3 in the pathogenesis of helminth-malaria concurrent infections.

\begin{abstract}
Abbreviations
ALT: Alanine aminotransferase; AST: Aminotransferase; CCL: C-C motif chemokine ligand; ECP: Eosinophil cationic protein; EPX: Eosinophil protein X; Gal: Galectin; H\&E: Hematoxylin and eosin; IFNy: Interferon-gamma; IL: Interleukin; iNOS: Inducible nitric oxide synthase; ip: Intraperitoneal; PbANKA: Plasmodium berghei of strain ANKA; PBS: Phosphate buffered solution; Pi: Postinfection; pRBCs: Parasitized red blood cells; qRT-PCR: Quantitative real-time reverse transcription polymerase chain reaction; SD: Standard deviation.
\end{abstract}

\section{Acknowledgments}

Not applicable.

\section{Authors' contributions}

FL designed the experiments, analyzed the data, wrote and edited the manuscript. XM and ZY conducted the experiments and analyzed the data. YC conducted the experiments. SH and JS edited the manuscript. All authors read and approved the final manuscript.

\section{Funding}

This study was supported by the Natural Science Foundation of China (nos. 81971955, 81873218, and 81471973), the Natural Science Foundation of Guangdong Province, China (no. 2019A1515011667), the open project of Key Laboratory of Tropical Disease Control of Ministry of Education, Sun Yat-sen University, China (no. 2020ZX02), the undergraduate teaching quality engineering and teaching reform project of Sun Yat-sen University, China (no. 2020/72), the National Science and Technology Major Project of Ministry of Science and Technology of China (no. 2018ZX09303008), and the research project of Guangdong Administration of Traditional Chinese medicine, China (no. 2019/43).

\section{Availability of data and materials}

Data supporting the conclusions of this article are included within the article.

\section{Ethics approval and consent to participate}

This study was carried out on the basis of National Guidelines for Experimental Animal Welfare (Ministry of Science and Technology of China, 2006). Animal studies were conducted according to protocols approved by the Animal Experimentation Ethics Committee of Zhongshan School of Medicine on Laboratory Animal Care at Sun Yat-sen University, China (No. 2016-081).

\section{Consent for publication}

Not applicable.

\section{Competing interests}

The authors declare that they have no competing interests.

Received: 12 March 2020 Accepted: 24 August 2020

Published online: 03 September 2020

\section{References}

1. White NJ. The treatment of malaria. N Engl J Med. 1996;335:800-6.

2. Ateba-Ngoa U, Adegnika AA, Zinsou JF, Kassa Kassa RF, Smits H, MassingaLoembe $M$, et al. Cytokine and chemokine profile of the innate and adaptive immune response of Schistosoma haematobium and Plasmodium falciparum single and co-infected school-aged children from an endemic area of Lambaréné, Gabon. Malar J. 2015;14:94.

3. Bethony J, Brooker S, Albonico M, Geiger SM, Loukas A, Diemert D, et al. Soil-transmitted helminth infections: ascariasis, trichuriasis, and hookworm. Lancet. 2006;367:1521-32.

4. Mulu A, Legesse M, Erko B, Belyhun Y, Nugussie D, Shimelis T, et al. Epidemiological and clinical correlates of malaria-helminth co-infections in southern Ethiopia. Malar J. 2013;12:227.

5. Sokhna C, Le Hesran JY, Mbaye PA, Akiana J, Camara P, Diop M, et al. Increase of malaria attacks among children presenting concomitant infection by Schistosoma mansoni in Senegal. Malar J. 2004;3:43.

6. Nacher M, Gay F, Singhasivanon P, Krudsood S, Treeprasertsuk S, Mazier $\mathrm{D}$, et al. Ascaris lumbricoides infection is associated with protection from cerebral malaria. Parasite Immunol. 2000;22:107-13.

7. Brutus L, Watier L, Hanitrasoamampionona V, Razanatsoarilala H, Cot M. Confirmation of the protective effect of Ascaris lumbricoides on Plasmodium falciparum infection: results of a randomized trial in Madagascar. Am J Trop Med Hyg. 2007;77:1091-5.

8. Degarege A, Animut A, Legesse M, Erko B. Malaria severity status in patients with soil-transmitted helminth infections. Acta Trop. 2009;112:8-11.

9. Oboth P, Gavamukulya Y, Barugahare BJ. Prevalence and clinical outcomes of Plasmodium falciparum and intestinal parasitic infections among children in Kiryandongo refugee camp, mid-western Uganda: a cross sectional study. BMC Infect Dis. 2019;19:295.

10. Babamale OA, Ugbomoiko US, Heukelbach J. High prevalence of Plasmodium falciparum and soil-transmitted helminth co-infections in 
a periurban community in Kwara State, Nigeria. J Infect Public Health. 2018;11:48-53.

11. Liao C, Cheng X, Liu M, Wang X, Boireau P. Trichinella spiralis and tumors: cause, coincidence or treatment? Anticancer Agents Med Chem. 2018;18:1091-9.

12. Pozio E, Hoberg E, La Rosa G, Zarlenga DS. Molecular taxonomy, phylogeny and biogeography of nematodes belonging to the Trichinella genus. Infect Genet Evol. 2009;9:606-16.

13. Mukaratirwa S, La Grange L, Pfukenyi DM. Trichinella infections in animals and humans in sub-Saharan Africa: a review. Acta Trop. 2013;125:82-9.

14. Collins SM, Blennerhassett PA, Blennerhassett MG, Vermillion DL. Impaired acetylcholine release from the myenteric plexus of Trichinellainfected rats. Am J Physiol. 1989;257:G898-903.

15. Ding J, Bai $X$, Wang X, Shi H, Cai X, Luo X, et al. Immune cell responses and cytokine profile in intestines of mice infected with Trichinella spiralis. Front Microbiol. 2017;8:2069.

16. Fabre V, Beiting DP, Bliss SK, Gebreselassie NG, Gagliardo LF, Lee NA, et al. Eosinophil deficiency compromises parasite survival in chronic nematode infection. J Immunol. 2009;182:1577-83.

17. Bailenger J, Lucchese F, Peychaud A, Haumont G, Cabannes A. Inhibition of Plasmodium berghei in rats infested with Strongyloides ratti or Trichinella spiralis; role of high blood corticosterone in reaction to the development of helminths. Ann Parasitol Hum Comp. 1985;60:435-43.

18. Ngwenya BZ. Enhanced resistance to Plasmodium berghei in mice previously infected with Trichinella spiralis. Parasite Immunol. 1982:4:197-207.

19. Li F, Cui J, Wang Z-Q, Jiang P. Sensitivity and optimization of artificial digestion in the inspection of meat for Trichinella spiralis. Foodborne Pathog Dis. 2010;7:879-85.

20. Liu J, Huang S, Su XZ, Song J, Lu F. Blockage of galectin-receptor interactions by a-lactose exacerbates Plasmodium berghei-induced pulmonary immunopathology. Sci Rep. 2016;6:32024.

21. Fazalul Rahiman SS, Basir R, Talib H, Tie TH, Chuah YK, Jabbarzare M, et al. Interleukin-27 exhibited anti-inflammatory activity during Plasmodium berghei infection in mice. Trop Biomed. 2013;30:663-80.

22. Viriyavejakul $P$, Khachonsaksumet $V$, Punsawad $C$. Liver changes in severe Plasmodium falciparum malaria: histopathology, apoptosis and nuclear factor kappa B expression. Malar J. 2014;13:106.

23. Jain A, Kaushik R, Kaushik RM. Malarial hepatopathy: clinical profile and association with other malarial complications. Acta Trop. 2016;159:95-105.

24. Maizels RM, Balic A, Gomez-Escobar N, Nair M, Taylor MD, Allen JE. Helminth parasites - masters of regulation. Immunol Rev. 2004;201:89-116.

25. Nacher M, Singhasivanon P, Gay F, Silachomroon U, Phumratanaprapin W, Looareesuwan S. Contemporaneous and successive mixed Plasmodium falciparum and Plasmodium vivax infections are associated with Ascaris lumbricoides: an immunomodulating effect? J Parasitol. 2001;87:912-5.

26. Ashour DS. Trichinella spiralis immunomodulation: an interactive multifactorial process. Expert Rev Clin Immunol. 2013;9:669-75.

27. Cypess RH, Lubiniecki AS, Swidwa DM. Decreased susceptibility to Listeria monocytogenes in mice after infection with Trichinella spiralis. Infect Immun. 1974;9:477-9.

28. Lemaitre M, Watier L, Briand V, Garcia A, Le Hesran JY, Cot M. Coinfection with Plasmodium falciparum and Schistosoma haematobium: additional evidence of the protective effect of schistosomiasis on malaria in Senegalese children. Am J Trop Med Hyg. 2014;90:329-34.

29. Helmby H, Kullberg M, Troye-Blomberg M. Altered immune responses in mice with concomitant Schistosoma mansoni and Plasmodium chabaudi infections. Infect Immun. 1998;66:5167-74.

30. Mathews SE, Bhagwati MM, Agnihotri V. Clinical spectrum of infection, from benign to severe malaria: a tertiary care prospective study in adults from Delhi, India. Trop Parasitol. 2019;9:88-92.

31. Walters JH, McGREGOR IA. The mechanism of malarial hepatomegaly and its relationship to hepatic fibrosis. Trans R Soc Trop Med Hyg. 1960;54:135-45.

32. Aikawa M, Suzuki M, Gutierrez Y. Pathology of malaria. In: Kreier JP, editor. malaria. New York: Academic Press; 1980. p. 47-102.

33. Wilson S, Jones FM, Mwatha JK, Kimani G, Booth M, Kariuki HC, et al. Hepatosplenomegaly is associated with low regulatory and Th2 responses to schistosome antigens in childhood schistosomiasis and malaria coinfection. Infect Immun. 2008;76:2212-8.

34. Wilson S, Jones FM, Mwatha JK, Kimani G, Booth M, Kariuki HC, et al. Hepatosplenomegaly associated with chronic malaria exposure: evidence for a pro-inflammatory mechanism exacerbated by schistosomiasis. Parasite Immunol. 2009;31:64-71.

35. Sangweme D, Shiff C, Kumar N. Plasmodium yoelii: adverse outcome of non-lethal P. yoelii malaria during co-infection with Schistosoma mansoni in BALB/c mouse model. Exp Parasitol. 2009;122:254-9.

36. Ozer J, Ratner M, Shaw M, Bailey W, Schomaker S. The current state of serum biomarkers of hepatotoxicity. Toxicology. 2008;245:194-205.

37. Bowdridge $S$, Gause WC. Regulation of alternative macrophage activation by chromatin remodeling. Nat Immunol. 2010;11:879-81.

38. Bhattacharya S, Aggarwal A. M2 macrophages and their role in rheumatic diseases. Rheumatol Int. 2019;39:769-80.

39. Kang SA, Park MK, Park SK, Choi JH, Lee DI, Song SM, et al. Adoptive transfer of Trichinella spiralis-activated macrophages can ameliorate both Th1and Th2-activated inflammation in murine models. Sci Rep. 2019;9:6547.

40. Craig JM, Scott AL. Antecedent Nippostrongylus infection alters the lung immune response to Plasmodium berghei. Parasite Immunol. 2017. https ://doi.org/10.1111/pim.12441.

41. Nobes MS, Ghabrial H, Simms KM, Smallwood RB, Morgan DJ, Sewell RB. Hepatic Kupffer cell phagocytotic function in rats with erythrocytic-stage malaria. J Gastroenterol Hepatol. 2002;17:598-605.

42. Bellows CF, Molina RM, Brain JD. Diminished organelle motion in murine Kupffer cells during the erythrocytic stage of malaria. J R Soc Interface. 2011:8:711-9.

43. MacKinnon AC, Farnworth SL, Hodkinson PS, Henderson NC, Atkinson $\mathrm{KM}$, Leffler $\mathrm{H}$, et al. Regulation of alternative macrophage activation by galectin-3. J Immunol. 2008;180:2650-8.

44. Kho S, Minigo G, Andries B, Leonardo L, Prayoga P, Poespoprodjo JR, et al. Circulating neutrophil extracellular traps and neutrophil activation are increased in proportion to disease severity in human malaria. J Infect Dis. 2019;219:1994-2004

45. Chen $L$, Zhang Z, Sendo F. Neutrophils play a critical role in the pathogenesis of experimental cerebral malaria. Clin Exp Immunol. 2000;120:125-33.

46. Sercundes MK, Ortolan LS, Debone D, Soeiro-Pereira PV, Gomes E, Aitken EH, et al. Targeting Neutrophils to prevent malaria-associated acute lung injury/acute respiratory distress syndrome in mice. PLoS Pathog. 2016;12:e1006054.

47. Nnalue NA, Friedman MJ. Evidence for a neutrophil-mediated protective response in malaria. Parasite Immunol. 1988;10:47-58.

48. Pierrot C, Adam E, Hot D, Lafitte S, Capron M, George JD, et al. Contribution of T cells and neutrophils in protection of young susceptible rats from fatal experimental malaria. J Immunol. 2007;178:1713-22.

49. Bruschi F, Chiumiento L. Trichinella inflammatory myopathy: host or parasite strategy? Parasit Vectors. 2011;4:42.

50. Kurtzhals JA, Reimert CM, Tette E, Dunyo SK, Koram KA, Akanmori $\mathrm{BD}$, et al. Increased eosinophil activity in acute Plasmodium falciparum infection - association with cerebral malaria. Clin Exp Immunol. 1998;112:303-7.

51. Waters LS, Taverne J, Tai PC, Spry CJ, Targett GA, Playfair JH. Killing of Plasmodium falciparum by eosinophil secretory products. Infect Immun. 1987;55:877-81.

52. Durack DT, Ackerman SJ, Loegering DA, Gleich GJ. Purification of human eosinophil-derived neurotoxin. Proc Natl Acad Sci USA. 1981;78:5165-9.

53. Jose PJ, Griffiths-Johnson DA, Collins PD, Walsh DT, Mogbel R, Totty NF, et al. Eotaxin: a potent eosinophil chemoattractant cytokine detected in a guinea pig model of allergic airways inflammation. J Exp Med. 1994;179:881-7.

54. Forssmann U, Uguccioni M, Loetscher P, Dahinden CA, Langen $\mathrm{H}$, Thelen M, et al. Eotaxin-2, a novel CC chemokine that is selective for the chemokine receptor CCR3, and acts like eotaxin on human eosinophil and basophil leukocytes. J Exp Med. 1997;185:2171-6.

55. Shinkai A, Yoshisue H, Koike M, Shoji E, Nakagawa S, Saito A, et al. A novel human CC chemokine, eotaxin-3, which is expressed in IL-4-stimulated vascular endothelial cells, exhibits potent activity toward eosinophils. J Immunol. 1999;163:1602-10. 
56. Proctor WR, Chakraborty M, Chea LS, Morrison JC, Berkson JD, Semple K, et al. Eosinophils mediate the pathogenesis of halothane-induced liver injury in mice. Hepatology. 2013;57:2026-36.

57. Gebreselassie NG, Moorhead AR, Fabre V, Gagliardo LF, Lee NA, Lee JJ, et al. Eosinophils preserve parasitic nematode larvae by regulating local immunity. J Immunol. 2012;188:417-25.

58. Huang L, Gebreselassie NG, Gagliardo LF, Ruyechan MC, Luber KL, Lee $N A$, et al. Eosinophils mediate protective immunity against secondary nematode infection. J Immunol. 2015;194:283-90.

59. Bruschi F, Korenaga M, Watanabe N. Eosinophils and Trichinella infection: toxic for the parasite and the host? Trends Parasitol. 2008;24:462-7.

60. Liu FT, Rabinovich GA. Galectins as modulators of tumour progression. Nat Rev Cancer. 2005:5:29-41.

61. Young AR, Meeusen EN. Galectins in parasite infection and allergic inflammation. Glycoconj J. 2002;19:601-6.

62. Bunn PT, MontesdeOca M, Rivera FL, Kumar R, Edwards CL, Faleiro RJ, et al. Galectin-1 impairs the generation of anti-parasitic Th1 cell responses in the liver during experimental visceral leishmaniasis. Front Immunol. 2017;8:1307.

63. Auvynet C, Moreno S, Melchy E, Coronado-Martínez I, Montiel JL, Aguilar-Delfin I, et al. Galectin-1 promotes human neutrophil migration. Glycobiology. 2013;23:32-42.

64. Ge XN, Ha SG, Greenberg YG, Rao A, Bastan I, Blidner AG, et al. Regulation of eosinophilia and allergic airway inflammation by the glycan-binding protein galectin-1. Proc Natl Acad Sci USA. 2016;113:E4837-46.

65. Abebayehu D, Spence A, Boyan BD, Schwartz Z, Ryan JJ, McClure MJ. Galectin-1 promotes an $M 2$ macrophage response to polydioxanone scaffolds. J Biomed Mater Res A. 2017;105:2562-71.

66. Barrionuevo P, Beigier-Bompadre M, Ilarregui JM, Toscano MA, Bianco GA, Isturiz MA, et al. A novel function for galectin-1 at the crossroad of innate and adaptive immunity: galectin-1 regulates monocyte/macrophage physiology through a nonapoptotic ERK-dependent pathway. J Immunol. 2007:178:436-45.

67. Correa SG, Sotomayor CE, Aoki MP, Maldonado CA, Rabinovich GA. Opposite effects of galectin-1 on alternative metabolic pathways of L-arginine in resident, inflammatory, and activated macrophages. Glycobiology. 2003;13:119-28.
68. Poncini CV, Ilarregui JM, Batalla El, Engels S, Cerliani JP, Cucher MA, et al. Trypanosoma cruzi infection imparts a regulatory program in dendritic cells and T cells via galectin-1-dependent mechanisms. J Immunol. 2015;195:3311-24.

69. Liu FT, Hsu DK, Zuberi RI, Kuwabara I, Chi EY, Henderson WR. Expression and function of galectin-3, a beta-galactoside-binding lectin, in human monocytes and macrophages. Am J Pathol. 1995;147:1016-28.

70. Oakley MS, Majam V, Mahajan B, Gerald N, Anantharaman V, Ward JM, et al. Pathogenic roles of CD14, galectin-3, and OX40 during experimental cerebral malaria in mice. PLoS One. 2009;4:e6793.

71. Nieminen J, St-Pierre C, Sato S. Galectin-3 interacts with naive and primed neutrophils, inducing innate immune responses. J Leukoc Biol. 2005:78:1127-35.

72. Farnworth SL, Henderson NC, Mackinnon AC, Atkinson KM, Wilkinson T, Dhaliwal K, et al. Galectin-3 reduces the severity of pneumococcal pneumonia by augmenting neutrophil function. Am J Pathol. 2008;172:395-405.

73. Ge XN, Ha SG, Liu F-T, Rao SP, Sriramarao P. Eosinophil-expressed galectin-3 regulates cell trafficking and migration. Front Pharmacol. 2013;4:37.

74. Volarevic V, Markovic BS, Bojic S, Stojanovic M, Nilsson U, Leffler H, et al. Gal-3 regulates the capacity of dendritic cells to promote NKT-cellinduced liver injury. Eur J Immunol. 2015;45:531-43.

75. Petropolis DB, Faust DM, Deep Jhingan G, Guillen N. A new human 3D-liver model unravels the role of galectins in liver infection by the parasite Entamoeba histolytica. PLoS Pathog. 2014;10:e1004381.

76. Porcherie A, Mathieu C, Peronet R, Schneider E, Claver J, Commere P-H, et al. Critical role of the neutrophil-associated high-affinity receptor for lgE in the pathogenesis of experimental cerebral malaria. J Exp Med. 2011;208:2225-36.

77. Almkvist J, Karlsson A. Galectins as inflammatory mediators. Glycoconj J. 2002:19:575-81.

78. Uchino Y, Woodward AM, Mauris J, Peterson K, Verma P, Nilsson UJ, et al. Galectin-3 is an amplifier of the interleukin-1 beta-mediated inflammatory response in corneal keratinocytes. Immunology. 2018;154:490-9.

\section{Publisher's Note}

Springer Nature remains neutral with regard to jurisdictional claims in published maps and institutional affiliations.
Ready to submit your research? Choose BMC and benefit from:

- fast, convenient online submission

- thorough peer review by experienced researchers in your field

- rapid publication on acceptance

- support for research data, including large and complex data types

- gold Open Access which fosters wider collaboration and increased citations

- maximum visibility for your research: over $100 \mathrm{M}$ website views per year

At BMC, research is always in progress.

Learn more biomedcentral.com/submissions 